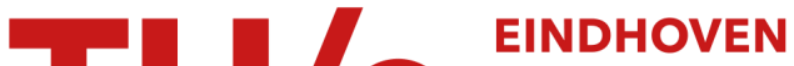

\section{Geometrically-shaped 64-point constellations via achievable information rates}

Citation for published version (APA):

Chen, B., Okonkwo, C., Lavery, D., \& Alvarado, A. (2018). Geometrically-shaped 64-point constellations via achievable information rates. In 20th International Conference on Transparent Optical Networks (ICTON 2018)

[Mo.B3.1] Institute of Electrical and Electronics Engineers. https://doi.org/10.1109/ICTON.2018.8473932

DOI:

10.1109/ICTON.2018.8473932

Document status and date:

Published: 26/09/2018

Document Version:

Accepted manuscript including changes made at the peer-review stage

Please check the document version of this publication:

- A submitted manuscript is the version of the article upon submission and before peer-review. There can be important differences between the submitted version and the official published version of record. People interested in the research are advised to contact the author for the final version of the publication, or visit the $\mathrm{DOI}$ to the publisher's website.

- The final author version and the galley proof are versions of the publication after peer review.

- The final published version features the final layout of the paper including the volume, issue and page numbers.

Link to publication

\section{General rights}

Copyright and moral rights for the publications made accessible in the public portal are retained by the authors and/or other copyright owners and it is a condition of accessing publications that users recognise and abide by the legal requirements associated with these rights.

- Users may download and print one copy of any publication from the public portal for the purpose of private study or research.

- You may not further distribute the material or use it for any profit-making activity or commercial gain

- You may freely distribute the URL identifying the publication in the public portal.

If the publication is distributed under the terms of Article $25 \mathrm{fa}$ of the Dutch Copyright Act, indicated by the "Taverne" license above, please follow below link for the End User Agreement:

www.tue.nl/taverne

Take down policy

If you believe that this document breaches copyright please contact us at:

openaccess@tue.nl

providing details and we will investigate your claim. 


\title{
Geometrically-shaped 64-point Constellations via Achievable Information Rates
}

\author{
Bin Chen ${ }^{1,2}$, Chigo Okonkwo ${ }^{1}$, Domaniç Lavery ${ }^{3}$, Alex Alvarado ${ }^{1}$ \\ ${ }^{1}$ Department of Electrical Engineering, Eindhoven University of Technology (TU/e), 5600MB Eindhoven, The Netherlands \\ ${ }^{2}$ School of Computer and Information, Hefei University of Technology (HFUT), Hefei, China \\ ${ }^{3}$ Department of Electronic and Electrical Engineering, University College London (UCL), London, WC1E 7JE, UK
}

\begin{abstract}
Achievable information rates (AIRs) are discussed as a performance metric to design optimized geometrically-shaped constellations. Mutual information and generalized mutual information are used to maximize the AIRs of 64-point constellations.
\end{abstract}

Keywords: Achievable information rates, coded modulation, geometric shaping, generalized mutual information, information theory, mutual information.

\section{InTRODUCTION}

Higher data rates to support the Internet's exponential traffic growth are required in currently deployed fibre optical communication systems [1]. One of the most established techniques for increasing spectral efficiency is through coded modulation, which is a combination of high-order modulation formats and forward error correction (FEC) [2]. In order to maintain reliable transmission and to approach the maximum transmission rate, a joint optimization of FEC and modulation formats is required. To estimate this rate, different metrics such as pre-FEC bit-error rate (BER), symbol error rate and Q-factor have been used in the optical communications community.

Achievable information rate (AIRs) are fast becoming popular metrics to measure the quality of the channel as well as its dependency on the choice of FEC and modulation formats. AIRs have been comprehensively studied in predicting the maximum throughput of a given channel, where the maximum reach has been evaluated for different modulation formats and FEC [3], [4]. Mutual information (MI) and generalized mutual information (GMI) are two AIR metrics for different coded modulation schemes. MI is the largest AIR for a symbol-wise decoder. Generalized mutual information (GMI) is an AIR for bit-interleaved coded modulation (BICM) [5], which neglects the conditional dependence among bits mapped to the same symbol.

To increase the data rate of the fibre channel, signal shaping has been investigated as a means to reduce the gap to the channel capacity. Two popular constellation shaping approaches are probabilistic shaping (PS) and geometric shaping (GS). The former changes the probabilities of the constellation points from a non-uniform distribution [6]-[10]. The latter employs non-equidistant constellation points [11]-[15]. PS has seen a significant interest in optical communications due to the its superior performance for a finite number of constellation points [5], [16]. However, low-complexity implementations of the required distribution matcher remain a challenge. On the other hand, GS has shown advantages when designing multi-dimensional [13] and nonlinearity tolerant [11], [14] modulation formats, as well as in the design of constellations for dispersion managed links [14], [15].

In this paper, we consider both MI and GMI metrics to design 64-ary constellations, with particular attention to GS. We examine the performance of numerically optimized GS and show that significant gains can be achieved compared with square QAM constellations. This analysis is done for both the additive white Gaussian noise (AWGN) channel and for the optical fiber channel. We also show that near-optimum performance can be achieved in a medium-haul link scenario if an adaptive distance-dependent modulator with only three GS modulation formats is used.

\section{ACHIEVABLE INFORMATION RATES}

The most commonly used channel for long-haul dispersion-unmanaged optical communications is the AWGN channel, which neglects the correlation caused by dispersion and nonlinear interference. The capacity of the AWGN channel under an average power constraint is $C=N / 2 \log _{2}(1+\mathrm{SNR})$. Here $N$ is the number of real dimensions and SNR is signal-to-noise ratio (SNR). In this paper, $N=4$ takes into account the two polarizations. The value of $C$ represents the maximum number of information bits per symbol that can be reliably transmitted through an AWGN channel.

For any memoryless channel with input $\boldsymbol{X}$ and output $\boldsymbol{Y}$, the largest achievable rate is the MI defined as

$$
\mathrm{MI}=I(\boldsymbol{X} ; \boldsymbol{Y}) \triangleq \mathbb{E}\left[\log _{2} \frac{f_{\boldsymbol{Y} \mid \boldsymbol{X}}(\boldsymbol{Y} \mid \boldsymbol{X})}{f_{\boldsymbol{Y}}(\boldsymbol{Y})}\right]
$$

where $\mathbb{E}$ denotes the expectation and $f_{\boldsymbol{Y} \mid \boldsymbol{X}}$ is the channel law. If bit-wise (BICM) decoders are used, i.e., the receiver is a soft demapper followed by a binary FEC decoder, the most popular AIR is the GMI. This GMI is the sum of $m$ bitwise MIs and is given by

$$
\mathrm{GMI} \triangleq \sum_{i=1}^{m} I\left(B_{i} ; \boldsymbol{Y}\right)=\sum_{i=1}^{m} \mathbb{E}\left[\log _{2} \frac{f_{\boldsymbol{Y} \mid B_{i}}\left(\boldsymbol{Y} \mid B_{i}\right)}{f_{\boldsymbol{Y}}(\boldsymbol{Y})}\right]
$$


where the bits mapped to the channel input $\boldsymbol{X}$ are represented by the random variables $B_{1}, B_{2}, \ldots, B_{m}$, and $m=$ $\log _{2} M$ is number of bits per constellation point. The channel capacity $C$ is lower-bounded via $C \geq \mathrm{MI} \geq \mathrm{GMI}$.

The GMI is usually considered as a performance metric for BICM systems, as they separate the detector from the decoder at the receiver. This allows the complexity of the receiver to be substantially reduced. However, an additional rate loss with respect to MI will be induced if no good binary labeling for the constellation is found.

\section{Geometric Constellation Shaping}

\subsection{AIR-based optimization problem}

From (1) it is clear that the MI depends on the channel SNR (denoted by $\gamma$ ) and the location of the constellation points $\mathcal{X}=\left\{\boldsymbol{X}_{1}, \boldsymbol{X}_{2}, \ldots, \boldsymbol{X}_{M}\right\}$ with $M=2^{m}$. The MI-based optimization problem can thus be formulated as:

$$
\mathcal{X}^{*}=\underset{\mathcal{X}}{\operatorname{argmax}}\{\operatorname{MI}(\gamma, \mathcal{X})\}, \quad \text { subject to : } \frac{1}{M} \sum_{X \in \mathcal{X}}\|\boldsymbol{X}\|^{2} \leq 1 .
$$

The GMI does not only depend on the location of the points and the SNR, but also on the binary labeling $\mathcal{L}$, which associate the signals to the bits at the input of the modulator through the one-to-one mapping $\mathcal{X} \rightarrow$ $\{0,1\}^{m}$. The optimization problem is therefore to find a constellation $\mathcal{X}^{*}$ and labeling $\mathcal{L}^{*}$ such that

$$
\left\{\mathcal{X}^{*}, \mathcal{L}^{*}\right\}=\underset{\mathcal{X}, \mathcal{L}}{\operatorname{argmax}}\{\operatorname{GMI}(\gamma, \mathcal{X}, \mathcal{L})\}, \quad \text { subject to }: \frac{1}{M} \sum_{X \in \mathcal{X}}\|\boldsymbol{X}\|^{2} \leq 1 .
$$

For both metrics above, the optimization in $\mathcal{X}$ is known to be difficult because MI and GMI usually have multiple local maxima. In this paper, we use the pairwise optimization algorithm from [20] to optimize $\mathcal{X}$ for MI. For the GMI optimization problem, we combine it with the binary switch algorithm [21] in an iterative manner. The optimization is repeated until the algorithm converges or a maximum number of iterations is reached.

Note that for small values of $M$, the constellations obtained with the iterative algorithm are independent of the initial constellation. However, initializing the algorithm from a known constellation, such as QAM or APSK, usually results in a significant reduction in the time to converge to the optimum constellation. Therefore, we chose the initial constellation to be QAM or APSK for the optimization process. In most cases, these two different initial constellations converge to the same optimum constellation.

\subsection{AWGN Results: AIRs and AIRs Gains}

In this section, we present the optimization results for constellations with 64 points. For comparison, we provide the results for MI-optimized and GMI-optimized GS constellations.
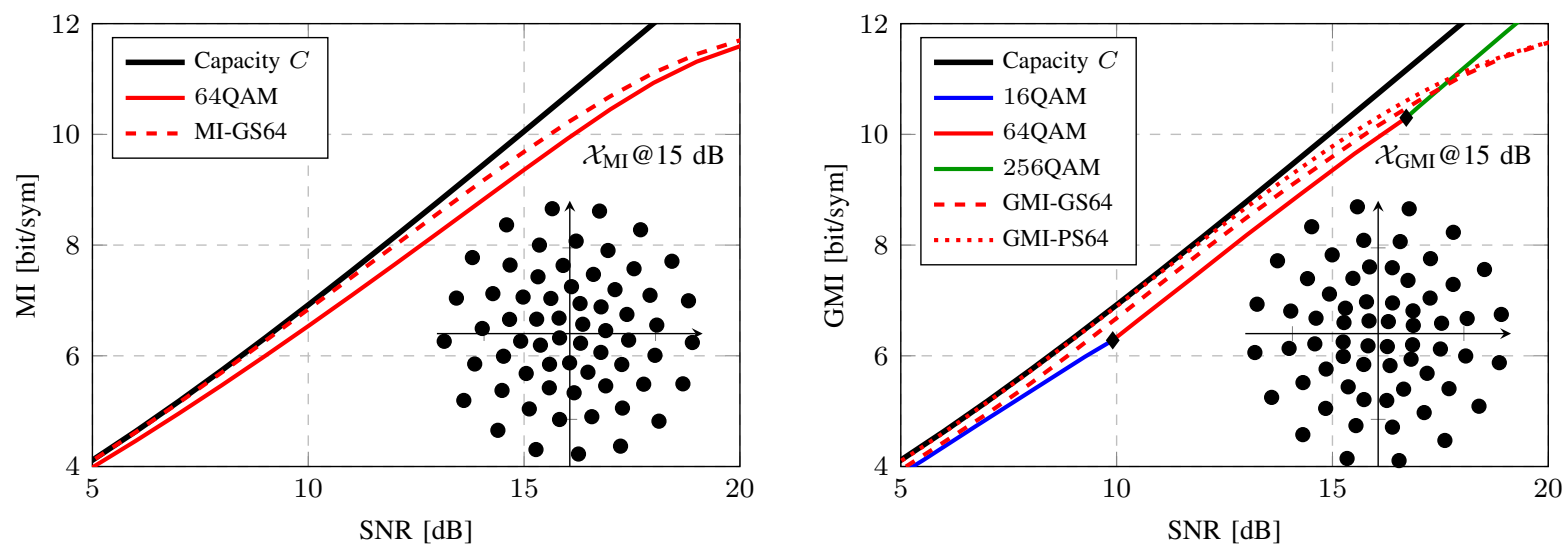

Figure 1: AIRs performance of geometrically shaped constellations. MI versus SNR for MI optimized formats (left) and GMI versus SNR for GMI optimized formats (right). The black diamonds indicates SNR values where the QAM cardinality should be changed. The channel capacity $C$ is shown for comparison. Insets: GS-optimized constellations for SNR=15 $\mathrm{dB}$. The baseline GMI of PS (red dotted line) from [8] is also shown.

In Fig. 1, we plot the MI and GMI of the optimized constellations for integer values of SNR $5 \leq \gamma \leq 20$ dB. In Fig. 1 (left), the red solid line shows the MI of 64QAM as baseline. The dashed line corresponds to MI of the optimized constellations. As it can be observed, the GS constellation can provide approximately $0.5 \mathrm{~dB}$ gain over 64QAM for AIR between 6 and 10 bits/4D-symbol. In Fig. 1 (right), we use the GMI of square QAM constellations as a baseline, which is known to cross each other at certain SNR values [4]. Fig. 1 (right) also shows the GMI performance of the GS and PS constellations. We can observe that both GS and PS can closely approach the AWGN capacity, and the loss due to the bit metric decoding is more evident for GS, which was also previously reported in [5], [16]. For AIR between 6 and 10 bits/4D-symbol, the gain of GS over 64QAM is approximately $0.4 \mathrm{~dB}$. Optimized constellations for SNR $\gamma=15 \mathrm{~dB}$ are shown as insets in Fig. 1. From these 
two constellations, we observe that the shape of the optimized constellation strongly depends on the chosen AIR metric. Note that the optimized constellations are different for each SNR.

To better quantify the throughput gains offered by GS constellations, we consider the AIR gains with respect to the MI of 64QAM and the GMI of 64QAM as the baseline for MI-optimized constellations and GMI-optimized constellations, respectively. These AIR gains are defined as $\delta_{\mathrm{MI}}=\mathrm{MI}^{*}-\mathrm{MI}^{Q A M}$ and $\delta_{\mathrm{GMI}}=\mathrm{GMI}^{*}-\mathrm{GMI}^{Q A M}$, where $\mathrm{MI}^{*}$ and $\mathrm{GMI}^{*}$ represent the AIRs obtained with the optimized constellations.

Fig. 2 (left) shows the obtained MI gains. The gray solid line is the MI gain with the optimized constellations for integer values of SNR $-5 \leq \gamma \leq 25 \mathrm{~dB}$. The colored dashed lines shows the MI gain for of a single constellation optimized for SNR $\gamma=13,15,17 \mathrm{~dB}$, respectively. We note from Fig. 2 (left) that the performance of the optimum GS constellation can be obtained by employing three 64-ary GS constellation formats in three SNR regimes, below $14 \mathrm{~dB}$ (blue), between $14 \mathrm{~dB}$ and $16 \mathrm{~dB}$ (red), and above $16 \mathrm{~dB}$ (green). We note that for MI optimized modulation, GS can achieve a gain of up to 0.35 bits/4D-symbol compared with 64QAM. In comparison, PS (dotted gray line) can achieve up to 0.45 bits/4D-symbol. As mentioned before, however, this gain come with a increased DSP complexity of both transmitter and receiver.
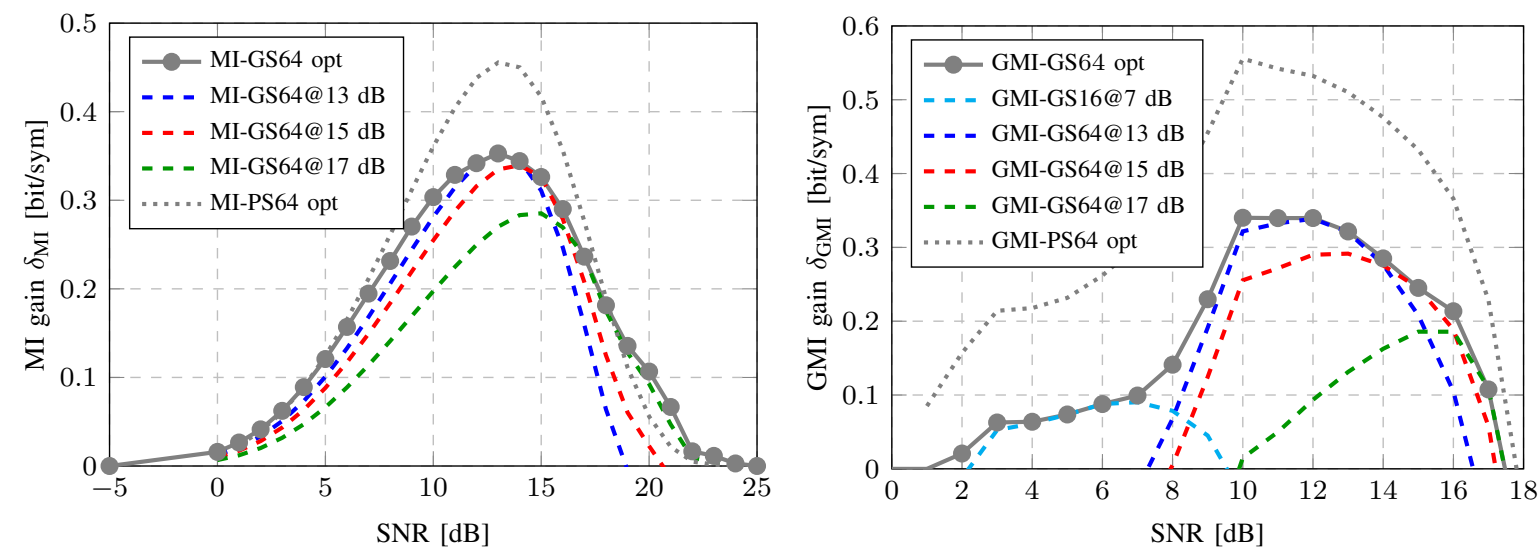

Figure 2: MI (left) and GMI gains (right) from geometrically shaped 64-ary constellations for all given SNRs (solid gray) and for a given SNR (dashed line). The baseline MI and GMI gains (gray dotted line) of PS from [7], [8] are also shown.

Fig. 2 (right) shows the obtained GMI gains. The gray solid line is the GMI gain with the optimized constellations for integer values of SNR $0 \leq \gamma \leq 18 \mathrm{~dB}$. The colored dashed lines show the GMI gain of a single constellation optimized for SNR $\gamma=13,15,17 \mathrm{~dB}$, respectively. We note from Fig. 2 (right) that the performance of the optimum GS constellation can be obtained by employing one 16-ary GS constellation and three 64-ary GS constellations in four SNR regimes: below $8 \mathrm{~dB}$ (cyan), $8 \mathrm{~dB}$ and $14 \mathrm{~dB}$ (blue), between 14 $\mathrm{dB}$ and $16 \mathrm{~dB}$ (red), and above $16 \mathrm{~dB}$ (green). GS constellations can achieve a gain of up to 0.35 bits/symbol compared with 64QAM. PS can achieve up to 0.55 bits/symbol.

\section{Numerical Analysis for a multi-Span Fiber Systems}

\subsection{Fiber Simulations}

We consider an optical fiber link comprising multiple standard single-mode fiber (SSMF) spans, amplified at the end of each span by an Erbium doped fiber amplifiers (EDFAs). The simulation parameters are given in Table I. Each WDM channel carries independent data and all of them are assumed to have the same transmitted power. At the receiver, an ideal receiver is used for detecting and chromatic dispersion is digitally compensated. Six GS constellations with 64 points-MI-optimized and GMI-optimized for SNR = 13, 15, $17 \mathrm{~dB}$-are simulated.

TABLE I: System parameters of the simulated multi-span optical link.

\begin{tabular}{|c|c|c|c|}
\hline \multicolumn{2}{|c|}{ Transmitter Parameters } & \multicolumn{2}{c|}{ Fiber Channel Parameters } \\
\hline Parameter Name & Value & Parameter Name & Value \\
\hline Wavelength-division multiplexing Channels & 11 & Attenuation & $0.2 \mathrm{~dB} / \mathrm{km}$ \\
\hline Symbol Rate & $45 \mathrm{Gbaud}$ & Dispersion Parameter & $17 \mathrm{ps} / \mathrm{nm} / \mathrm{km}$ \\
\hline Root-raised-cosine Roll-Off factor & 0.1 & Nonlinearity Parameter & $1.21 /(\mathrm{W} \cdot \mathrm{km})$ \\
\hline Channel Frequency Spacing & $50 \mathrm{GHz}$ & Fiber Span Length & $80 \mathrm{~km}$ \\
\hline Center Wavelength & $1550 \mathrm{~nm}$ & EDFA Noise Figure & $5 \mathrm{~dB}$ \\
\hline
\end{tabular}

\subsection{Simulation Results}

From the results in Sec. 3.2, we can predict that different GS constellations should be used for different ranges in transmission distance. In order to highlight the performance of each optimized modulation formats versus the transmission distance $L$, we show in Fig. 3 (left) the maximum MI by using three modulation formats optimized at different SNRs, which we denote by $\mathcal{X}_{\mathrm{SNR}}^{*}$. This maximum MI is then defined as $\mathrm{MI}^{*}(L)=$ $\max _{\mathrm{SNR} \in\{13,15,17\}} \operatorname{AIR}\left(L, \mathcal{X}_{\mathrm{SNR}}^{*}\right)$. The same is done for the GMI but taking the binary labeling into account.

We observe in Fig. 3 (left) that the GMI-optimized GS64 can achieve 9.6 bits/4D-symbol with $150 \mathrm{~km}$ increase in transmission distance, while the MI-optimized GS64 can further increase by an additional $100 \mathrm{~km}$. 
Fig. 3 (right) shows AIRs versus launch power per channel in $\mathrm{dBm}$ at $2400 \mathrm{~km}$ distance. GMI-optimized GS modulation for $\gamma=15 \mathrm{~dB}$, at the optimal launch power, can achieve a gain of 0.2 bits/symbol compared with 64QAM, while MI-optimized GS modulation for $\gamma=15 \mathrm{~dB}$ can achieve 0.33 bits/symbol. The measured channel SNRs after DSP for these modulations are around $14.5 \mathrm{~dB}$. Compared to the GMI gains achieved in AWGN channel (Fig. 2), the GMI gains achieved by PS and GS in the SSFM simulation are smaller, which is caused by the adverse effects of shaping in the presence of modulation-dependent nonlinear effect [8]. This negative effect is more evident for PS, which weakens its superior performance when compared to GS.
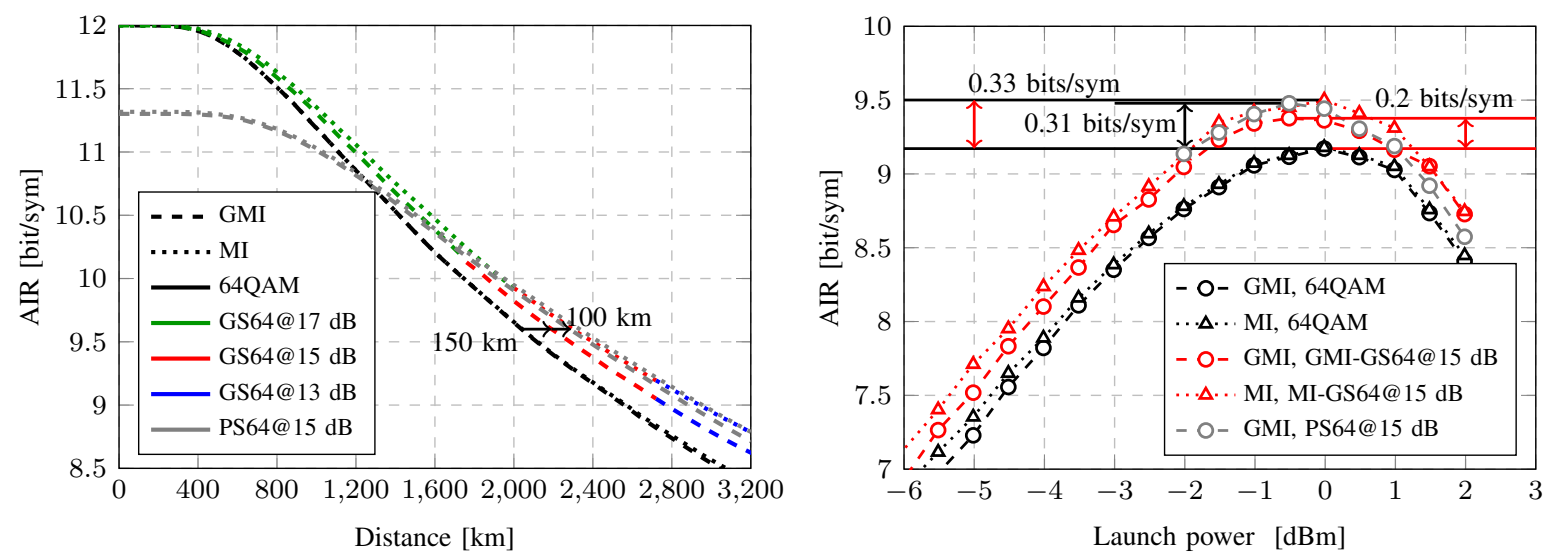

Figure 3: Left: AIRs as function of the transmission distance for different modulation formats. Right: AIRs as function of the transmitted power for different modulation formats over a $30 \times 80 \mathrm{~km} \mathrm{SSMF}$.

\section{Conclusions}

In this paper, we discussed the use of achievable information rates in the design fiber optical communication systems. It was shown that AIRs are efficient metrics for designing coded modulation schemes that use FEC and high-order modulation formats. The main focus of this paper was on design of optimized constellations and labeling to maximize the MI/GMI. For a medium-haul link, GS modulation formats could yield AIRs gains of up to $0.35 \mathrm{bits} / \mathrm{symbol}$, and a reach extension of up to $250 \mathrm{~km}(\approx+12 \%)$. In combination with FEC, these constellations are expected to be very effective for spectrally-efficient optical transmissions.

Acknowledgements: The authors would like to thank Dr. Tobias Fehenberger (Technical University of Munich) for providing some of the data in Fig. 2 (previously published in [7], [8]). The work of B. Chen is partially supported by the National Natural Science Foundation of China (NSFC) under Grant 61701155. The work of A. Alvarado is partially supported by the Netherlands Organisation for Scientific Research (NWO) via the VIDI Grant ICONIC (project number 15685) and by the European Research Council (ERC) under the European Unions Horizon 2020 research and innovation programme (grant agreement No 757791). The work of C. Okonkwo is partially supported by the EC through the H2020 project Metro-Haul (Grant Agreement number 761727). D. Lavery is supported by the Royal Academy of Engineering through the research fellowships programme.

\section{REFERENCES}

[1] D. J. Richardson, "Filling The Light Pipe," Science, vol. 330, 6002, 327328 (2010).

[2] G. Ungerboeck, "Channel Coding with Multilevel/Phase Signals," IEEE Trans. Inf. Theo. 28, 55-67 (1982).

[3] G. Liga et al., "Information Rates of Next-generation Long-haul Optical Fiber Systems using Coded Modulation,” J. Lightw. Technol., 35 (1), 113-123 (2017).

[4] A. Alvarado et al., "Achievable Information Rates for Fiber Optics: Applications and Computations,” J. Lightw. Technol., 36, 424-439 (2018).

[5] L. Szczecinski et al., Bit-Interleaved Coded Modulation: Fundamentals, Analysis and Design. John Wiley \& Sons, 2015.

[6] F. Buchali et al., "Rate Adaptation and Reach Increase by Probabilistically Shaped 64-QAM: an Experimental Demonstration," J. Lightw. Technol., Vol. 34, 1599-1609 (2016).

[7] T. Fehenberger et al., "Sensitivity Gains by Mismatched Probabilistic Shaping for Optical Communication Systems," IEEE Photon. Technol. Lett., Vol. 28 (7), p. 786 (2016).

[8] T. Fehenberger et al., "On Probabilistic Shaping of Quadrature Amplitude Modulation for the Nonlinear Fiber Channel," J. Lightw. Technol., Vol. 34, 5063-5073 (2016).

[9] G. Bocherer et al., "Fast Probabilistic Shaping Implementation for Long-Haul Fiber-Optic Communication Systems," Proc. ECOC, Tu.2.D.3 (2017).

[10] R. Maher et al., "Constellation Shaped 66 GBd DP-1024QAM Transceiver with 400 km Transmission over Standard SMF," Proc. ECOC, Th.PDP.B.2 (2017).

[11] Z. Qu et al., "Geometrically Shaped 16QAM Outperforming Probabilistically Shaped 16QAM," Proc. ECOC, Th.2.F.4 (2017).

[12] S. Zhang et al., "Design and Comparison of Advanced Modulation Formats Based on Generalized Mutual Information," J. Lightw. Technol., Vol. 36, 416-423 (2018).

[13] A. I. A. El-Rahman et al., "Multidimensional Geometric Shaping for QAM Constellations," Proc. ECOC, Tu.1.D.3 (2017).

[14] K. Kojima et al., "Nonlinearity-Tolerant Four-Dimensional 2A8PSK Family for 5-7 Bits/Symbol Spectral Efficiency," J. Lightw. Technol., Vol. 35, 1383-1391, (2017).

[15] D. S. Millar et al., "Coded Modulation for Next-Generation Optical Communications," Proc. OFC, Tu3C.3 (2018)

[16] F. Steiner et al., "Comparison of Geometric and Probabilistic Shaping with Application to ATSC 3.0," Proc. SCC, (2017).

[17] L. Schmalen et al., "Performance Prediction of Nonbinary Forward Error Correction in Optical Transmission Xxperiments," J. Lightw. Technol, vol. 35, no. 4, pp. 1015-1026, (2017).

[18] X. Li et al., "Bit-interleaved Coded Modulation with Iterative Decoding using Soft Feedback," Electron. Lett., vol. 34, no. 10, pp. 942-943, (1998).

[19] U. Wachsmann et al., "Multilevel codes: Theoretical Concepts and Practical Design Rules," IEEE Trans. Inf. Theory, vol. 45, no. 5, pp. 13611391, (1999).

[20] B. Moore et al., "Pairwise Optimization of Modulation Constellations for Non-uniform Sources," IEEE Can. J. Elect. Comput. Eng., Vol. 34 (4), (2009).

[21] F. Schreckenbach et al., "Optimization of Symbol Mappings for Bit-Interleaved Coded Modulation with Iterative Decoding," IEEE Commun. Lett., Vol. 7 (12), p. 593 (2003). 